\title{
ANÁLISE DA SENSIBILIDADE DOS COMPONENTES DA EQUAÇÃO DE HARGREAVES - SAMANI PARA A REGIÃO DE BEBEDOURO - SP
}

\section{LUIZ FABIANO PALARETTI ${ }^{1}$; EVERARDO CHARTUNI MANTOVANI ${ }^{2}$; GILBERTO CHOHAKU SEDIYAMA $^{2}$}

\author{
${ }^{1}$ Universidade Estadual Paulista (UNESP), Faculdade de Ciência Agrárias e Veterinárias, Departamento de \\ Engenharia Rural, Jaboticabal, SP, Brasil \\ ${ }^{2}$ Universidade Federal de Viçosa, Departamento de Engenharia Agrícola, Viçosa, MG, Brasil \\ lpalaretti@fcav.unesp.br, everardo@ufv.br, g.sediyama@ufv.br
}

Recebido Junho de 2012 - Aceito Agosto de 2013

\begin{abstract}
RESUMO
A estimativa da evapotranspiração de referência (ETo) subsidia os cálculos de consumo de água na agricultura irrigada. O uso de metodologias mais simples são comuns, porém devem ser criteriosamente estudadas. No presente estudo avaliou-se a sensibilidade da equação de Hargreaves - Samani frente ao padrão Penman-Monteith - FAO, na estimativa da ETo, para as condições climáticas da região de Bebedouro-SP, simulando os efeitos de acréscimos e decréscimos de $5 \%$ nas temperaturas, resultando em dez cenários distintos (PM - Penman-Montheit - FAO; S0 - Hargreaves Samani original; S1 à S8 - Hargreaves Samani - com alterações). Os resultados foram comparados em sensibilidade, por regressão linear, precisão e o índice de concordância. Conclui-se que a equação de Hargreaves Samani superestima valores mensais de ETo em $13 \%$ comparado ao método padrão. Uma variação de $5 \%$ nas temperaturas máximas e mínimas resultaram em erros de até $1 \mathrm{~mm} \mathrm{dia}^{-1}$, sendo o acréscimo mais expressivo na temperatura máxima.
\end{abstract}

Palavras-chave: irrigação, consumo de água, Penman-Monteith, citros

\begin{abstract}
SENSITIVITY ANALYSIS OF HARGREAVES SAMANI EQUATION COMPONENTS FOR BEBEDOURO REGION - SP
\end{abstract}

The estimation of reference evapotranspiration (ETo) subsidizes the calculations of water consumption in irrigated agriculture. The use of simpler methodologies are common however should be carefully studied. The present study evaluated the sensitivity of the Hargreaves - Samani equation compared to the standard Penman-Monteith - FAO to estimate ETo for the climatic conditions of the BebedouroSP region, simulating the effects of increasing and decreasing $5 \%$ in the temperatures, resulting in ten different scenarios (PM - Penman-Montheit - FAO; S0 - original Hargreaves Samani; S1 to S8 - modified Hargreaves Samani). The obtained results of sensitivity, linear regression, accuracy and concordance index, were compared. We concluded that the Hargreaves - Samani equation overestimates the monthly values of the ETo in $13 \%$ compared to the standard method. A variation of $5 \%$ in maximum and minimum temperatures resulted in errors of up to $1 \mathrm{~mm} \mathrm{day}^{-1}$ (monthly average) and this increase is more expressive in maximum temperature.

Keywords: irrigation, water consumption, Penman-Monteith, citrus crop. 


\section{INTRODUÇÃO}

A evapotranspiração, considerada como uso consuntivo de água, é um componente do ciclo hidrológico, que contabiliza, o somatório da evaporação de água do solo e da transpiração vegetal.

Os métodos de estimativa da evapotranspiração são agrupados em categorias: (i) empíricos; (ii) aerodinâmico; (iii) balanço de energia; (iv) combinados; (v) correlação dos turbilhões (Pereira et al., 1997).

A metodologia padrão para estimativa da evapotranspiração de referência (ETo), envolve a interação entre o processo físico de perda de água, parâmetros fisiológicos e aerodinâmicos da planta. A proposta de Penman-Monteith considera uma superfície coberta por vegetação hipotética com $12 \mathrm{~cm}$ de altura, resistência aerodinâmica da superfície

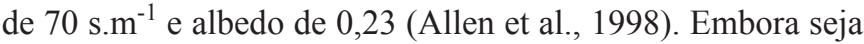
considerado o método com melhor desempenho na estimativa, apresenta restrições quanto à grande quantidade de informações necessárias para tal. Sendo que a maioria destas, principalmente relacionadas ao clima, nem sempre estão disponíveis regionalmente, em quantidade e qualidade.

É de fundamental importância para a região de Bebedouro - SP, pela sua expressividade no cenário citrícola nacional, a determinação correta da ETo, uma vez que a irrigação de citros, nesta região, evoluiu em sistemas e áreas. Estima-se que $26,6 \%$ dos pomares Brasileiros (FNP, 2011; IBGE, 2006) sejam irrigados, logo, incertezas nos valores de ETo refletem-se em maiores custos e riscos, podendo afetar negativamente a rentabilidade do setor.

Dessa forma, é pertinente salientar que a escolha das informações utilizadas na estimativa do consumo de água pelas plantas deve ser criteriosa, evitando-se generalizações quanto ao uso das diversas metodologias, sob todas as condições, devendo-se considerar as interações de processos descritos na literatura clássica (Pereira et al., 1997).

O método de estimativa da ETo proposto por Hargreaves e Samani (1985) é considerado empírico (Pereira et al., 1997) e convenientemente requer uma menor quantidade de informações meteorológicas para tal. Indicado para estimativa em períodos superiores a 7 - 10 dias (Mendonça et al., 2003), este apresenta tendência a superestimativa dos valores de ETo em relação ao método padrão (Batista et al. 2007), e subestimativa em relação ao lisímetro (Souza et al. 2011), requerendo uma calibração dos coeficientes da equação, regionalmente, objetivando atenuar essa discrepância (Vega e Jara, 2009).

A calibração é feita por regressão linear, considerando uma variável dependente em função de uma independente. Uma vez encontrado o valor da variável dependente, estima-se o da independente (França Neto, 2003). O método padrão é utilizado para tal finalidade onde se geram coeficientes de ajustes para aproximação dos valores do método empírico.

Após a definição dos parâmetros de calibração da equação, aliado ao fato de possíveis erros dos dados meteorológicos utilizados para determinação da ETo, recomenda-se a realização de uma análise de sensibilidade visando a explicitação da influência e dimensão desta, nos distintos componentes da equação de estimativa (Mello, 1998). Dessa forma, gera-se um coeficiente de sensibilidade de uma variável dependente em função de uma independente da equação, permitindo conhecer qual o efeito do erro no resultado final.

Um dos objetivos fundamentais de uma análise de sensibilidade é mostrar qual alcance de precisão é requerido para a introdução de uma variável ou de um componente no modelo. Consequentemente, onde será exigido mais critério na calibração do método, uma vez que, cada componente o influencia de forma diferenciada.

O presente estudo teve como objetivo analisar a sensibilidade dos parâmetros: temperaturas máxima e mínima na metodologia proposta por Hargreaves e Samani (1985), para estimativa da evapotranspiração de referência (ETo).

\section{DADOS E METODOLOGIA}

O estudo foi realizado na região norte do estado de São Paulo. Considerou-se a cidade de Bebedouro, localizada nas coordenadas geográficas: latitude $20^{\circ} 53^{\prime} 16^{\prime \prime}$ 'S, longitude $48^{\circ} 28^{\prime} 11^{\prime \prime} \mathrm{W}$ e altitude $601 \mathrm{~m}$, como representante regional.

O clima da região é do tipo Aw, segundo classificação climática de Köppen modificada (Rolim et al. 2007), com médias anuais de temperatura mínima, máxima e precipitação de $17,5^{\circ} \mathrm{C}$ e $31{ }^{\circ} \mathrm{C} 1.440 \mathrm{~mm}$, respectivamente.

$\mathrm{Na}$ estimativa dos valores de evapotranspiração de referência (ETo) foram utilizadas duas metodologias, escolhidas criteriosamente em função da quantidade de variáveis climatológicas requeridas para as estimativas. A metodologia de referência foi a proposta por Penman-Monteith (Allen et al., 1998) (Equação 1) e a outra por Hargreaves e Samani (Equação 2).

$$
E T_{0}=\left[\frac{0,408(R n-G)+\gamma \frac{900}{T+273} U_{2} \cdot\left(e_{s}-e_{a}\right)}{\Delta+\gamma\left(1+0,34 U_{2}\right)}\right] \times 0.408
$$

em que, ETo: evapotranspiração de referência $\left(\mathrm{mm} \mathrm{dia}^{-1}\right)$; Rn: saldo de radiação à superfície $\left(\mathrm{MJ} \mathrm{m}^{-2} \mathrm{~d}^{-1}\right) ; \mathrm{T}$ : temperatura do ar a $2 \mathrm{~m}$ de altura $\left({ }^{\circ} \mathrm{C}\right)$; G: fluxo de calor no solo $\left(\mathrm{MJ} \mathrm{m}^{-2} \mathrm{~d}^{-1}\right)$;

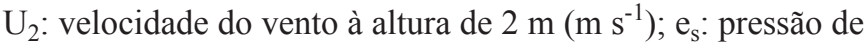
saturação de vapor $(\mathrm{kPa}) ; \mathrm{e}_{\mathrm{a}}$ : pressão de vapor atual do ar $(\mathrm{kPa})$; $\left(e_{s}-e_{a}\right)$ : déficit de pressão de vapor $(\mathrm{kPa}) ; \Delta$ : declividade da 
curva de pressão de vapor de saturação $\left(\mathrm{kPa}^{\circ} \mathrm{C}^{-1}\right)$ e $\gamma$ : coeficiente psicrométrico igual a $0,0622\left(\mathrm{kPa}^{\circ} \mathrm{C}^{-1}\right)$.

$$
\mathrm{ET}_{0}=0,0023 Q_{o}\left(T_{\text {máx }}-T_{\text {min }}\right)^{0,5}\left(T_{\text {méd }}+17,8\right)
$$

em que: $\mathrm{ET}_{0}$ : evapotranspiração de referência $\left(\mathrm{mm} \mathrm{d}^{-1}\right) ; \mathrm{Q}_{0}$ : radiação extraterrestre $\left(\mathrm{mm} \mathrm{d}^{-1}\right) ; \mathrm{T}_{\text {máx }}$ : temperatura máxima $\left({ }^{\circ} \mathrm{C}\right) ; \mathrm{T}_{\text {mín }}$ : temperatura mínima $\left({ }^{\circ} \mathrm{C}\right)$ e $\mathrm{T}_{\text {méd }}$ : temperatura média diária $\left({ }^{\circ} \mathrm{C}\right)$.

Os dados meteorológicos utilizados nas estimativas contemplam uma série histórica entre os anos de 1980 à 2000, registrada nas estações meteorológicas do Instituto Nacional de Meteorologia (INMET), localizadas nas cidades de Bebedouro, Colina e Barretos. Com base nos dados dessas três localidades geraram-se médias mensais das variáveis de temperatura.

Para dinamizar os cálculos de estimativas de evapotranspiração de referência, utilizou-se o software IRRIPLUS $^{\circledR}$. O software apresenta em seu conteúdo uma seção que permite a realização de simulações, requerendo dados meteorológicos médios diários específicos em função da equação utilizada.

Para o estudo dos efeitos da variação das temperaturas máxima $\left(\mathrm{T}_{\text {máx }}\right)$ e mínima $\left(\mathrm{T}_{\text {mín }}\right)$ na estimativa da ETo, simulou-se erros de cinco pontos percentuais. Os acréscimos e, ou decréscimos de 5\% foram aplicados nos valores das temperaturas fornecidas pelas estações meteorológicas. As variações nas temperaturas geraram 8 cenários (Tabela 1) e posteriormente estimados os valores de ETo utilizando as equações propostas.

Salienta-se que o estudo não contempla nenhuma alteração nas demais variáveis climáticas utilizadas nas estimativas da evapotranspiração de referência.

Quantitativamente gerou-se regressões lineares em função dos valores de ETo estimados pelos métodos, com relevância dos coeficientes linear $\left(\beta_{0}\right)$ e angular $\left(\beta_{l}\right)$ e seu respectivo $r^{2}$.

Calculou-se o erro padrão de estimativa (SEE) e o erro padrão ajustado (SEEa) (Jensen et al.,1990), o qual foi modificado por Mantovani (1993). Avaliou-se os cenários em função dos valores de estimativa do SEE e SEEa, para o maior valor do coeficiente de determinação $\left(\mathrm{r}^{2}\right)$ e da aproximação de zero do parâmetro $\beta_{0}$ e de um para os parâmetros $\beta_{1}$ e $\mathrm{r}^{2}$.

Calculou-se a precisão, dos valores de evapotranspiração de referência encontrados nos referidos cenários, segundo o valor do coeficiente de determinação, que indica o grau em

Tabela 1 - Cenários gerados pela simulação alterações provocadas nos valores de temperatura máxima e mínima, para cálculo da evapotranspiração de referências.

\begin{tabular}{ccc}
\hline Simulações & Máxima & Temperaturas \\
& Sem alteração & Sem alteração \\
\hline${ }^{2}$ SM 0 - HG & Sem alteração & Sem alteração \\
${ }^{3}$ S1 & $+5 \%$ & Sem alteração \\
S2 & $-5 \%$ & Sem alteração \\
S3 & Sem alteração & $+5 \%$ \\
S4 & Sem alteração & $-5 \%$ \\
S5 & $+5 \%$ & $+5 \%$ \\
S6 & $-5 \%$ & $-5 \%$ \\
S7 & $+5 \%$ & $-5 \%$ \\
S8 & $-5 \%$ & $+5 \%$ \\
\hline
\end{tabular}

*PM: Penman-Montheit; ${ }^{2} \mathrm{HG}$ : Método de Hargreaves-Samani; ${ }^{3} \mathrm{SX}$ : Hargreaves-Samani - Alterado. 
que a regressão explica a soma do quadrado total e do índice de concordância ("d"), que expressa a aproximação dos valores de ETo estimados pelas combinações em relação ao estimado pelo padrão (Willmott et al. 1985), (Equação 3).

O índice de desempenho é distribuído nas seguintes classes: péssimo, $\mathrm{d} \leq 0,40$; mau, $0,41 \leq \mathrm{d} \leq 0,50$; sofrível, $0,51 \leq \mathrm{d} \leq 0,60$; mediano, $0,61 \leq \mathrm{d} \leq 0,65$; bom, $0,66 \leq \mathrm{d} \leq 0,75$; muito bom, $0,76 \leq \mathrm{d} \leq 0,85$; e ótimo, $\mathrm{d}>0,85$ (Camargo e Sentelhas, 1997).

$$
\mathrm{d}=1-\left[\frac{\Sigma(P i-O i)^{2}}{\Sigma(|P i-O|+|O i-O|)^{2}}\right]
$$

em que; Pi: valor estimado por Hargreaves-Samani e suas variações $\left(\mathrm{mm} \mathrm{dia}^{-1}\right)$; Oi: valor calculado pelo método de Penman-Monteih ( $\mathrm{mm} \mathrm{dia}^{-1}$ ) e $\mathrm{O}$ : média dos valores calculada pelo método de Penman-Monteih.

\section{RESULTADOS}

Na Figura 1 estão apresentados os valores de temperatura do ar, considerando a média mensal dos 20 anos de dados meteorológicos obtidos nas estações regionais. Observa-se que a temperatura média variou de $19,18{ }^{\circ} \mathrm{C}$ em julho a $25,69{ }^{\circ} \mathrm{C}$ em dezembro, alcançando valores extremos de $36,16^{\circ} \mathrm{C}$ em outubro e $10,59{ }^{\circ} \mathrm{C}$ em julho.

Os valores extremos médios da evapotranspiração variaram de 2,92 à 7,48 $\mathrm{mm} \mathrm{dia}^{-1}$ observados no método padrão (PM) e em S7, respectivamente (Figura 2). O comportamento observado em S7 é atribuído a redução de 5\% na temperatura mínima, evidenciando o efeito notório da temperatura máxima na estimativa da evapotranspiração pelo método de HargreavesSamani.
Observou-se superestimativa dos valores de ETo, em relação ao método padrão, em todos os cenários estudados, com valores relativos extremos de $1,81 \mathrm{~mm}$ em S8 e 22,82 $\mathrm{mm}$ em S7, respectivamente. Este comportamento demonstra a forte influência da temperatura máxima na estimativa da evapotranspiração por este método empírico (Tabela 2).

Observou-se que o desvio padrão dos valores de evapotranspiração de referência comporta-se como os da diferença relativa, com mínimo e máximo de 0,94 e 1,44 para os métodos de Penman-Monteith e S7, destacando o peso do componente $\mathrm{T}_{\max }$ na superestimativa dos valores de ETo.

O coeficiente de variação é considerado alto para todas as estimativas, sendo o menor valor encontrado em PM e o maior em S7, refletindo a condição apresentada para o desvio padrão de médias.

Quando o acréscimo de cinco pontos percentuais é sobre a temperatura mínima (S3), verifica-se que a superestimativa em relação ao padrão é menos expressiva, reduzindo a proporção quando $\mathrm{T}_{\text {máx }}$ é reduzida conjuntamente (S6). Comparando $\mathrm{S} 6$ e S8 observa-se o peso da influência de $\mathrm{T}_{\max }$ em $\mathrm{T}_{\min }$ provavelmente, por efeito da temperatura média.

Analisando-se a variação de lâminas, que representa a diferença entre os valores de ETo média estimada no cenário e o obtido pela equação de Penman_Montheit, observa-se que o maior valor foi próximo a um (S7). O valor de $1,03 \mathrm{~mm} \mathrm{dia}^{-1}$ representa baixa expressividade no consumo de água pelas plantas, uma vez que em condições de irrigação em campo, erros dessa magnitude durante a aplicação são praticamente desprezíveis.

As Figuras 3 e 4 indicam correlação semelhante entre os dados diários da evapotranspiração de referência estimado pelo método de Penman-Monteith - FAO e pelo método proposto por Hargreaves-Samani e as simulações, para a região de

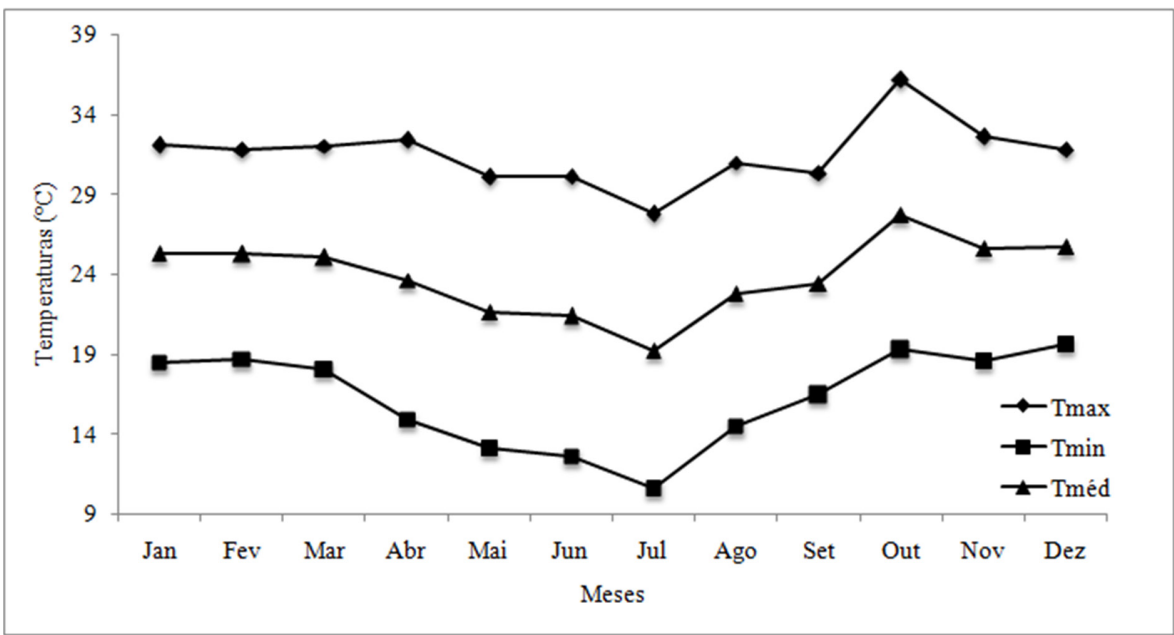

Figura 1 - Valores históricos de temperatura do ar, média, mínima e máxima do ar, considerando 20 anos de dados observados em estações meteorológicas da região de Bebedouro-SP. 


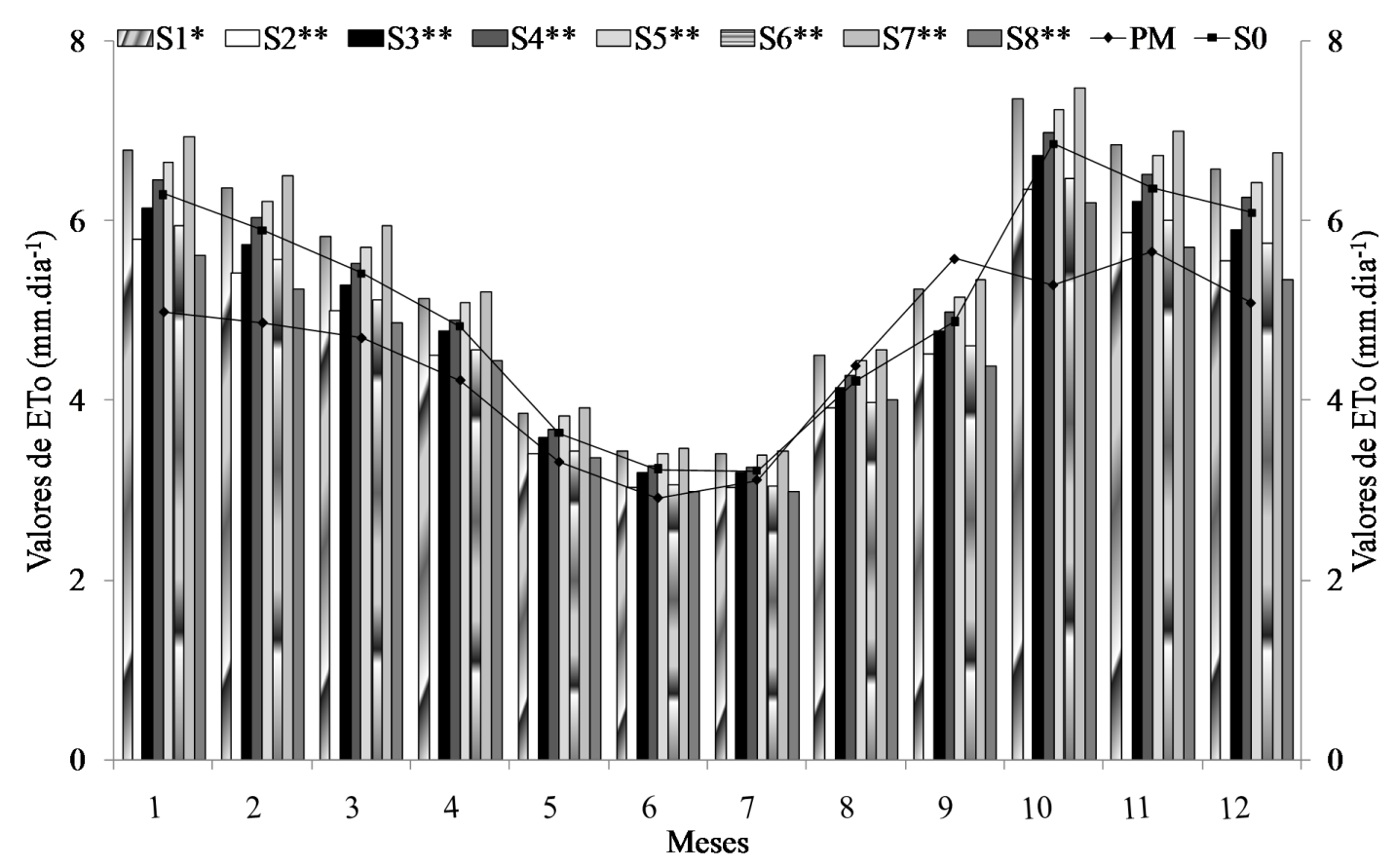

Figura 2 - Comportamento da ETo mensal, função dos métodos propostos e dos cenários simulados. Bebedouro - SP.

Tabela 2 - Valores das médias mensais da ETo determinadas pelos métodos comparados, em mm, para Bebedouro, SP.

\begin{tabular}{ccccccccccc}
\hline Parâmetros & PM & S0 & S1 & S2 & S3 & S4 & S5 & S6 & S7 & S8 \\
\hline \hline Média - PM & 4,51 & 5,08 & 5,46 & 4,70 & 4,97 & 5,18 & 5,36 & 4,81 & 5,54 & 4,60 \\
Dif. Relativa & $\%$ & 12,57 & 20,86 & 4,14 & 10,19 & 14,7 & 18,67 & 6,54 & $\mathbf{2 2 , 8 2}$ & $\mathbf{1 , 8 1}$ \\
Dif. Lâmina & - & 0,57 & 0,94 & 0,19 & 0,46 & 0,66 & 0,84 & 0,30 & 1,03 & 0,08 \\
Desvio Padrão & 0,94 & 1,28 & 1,40 & 1,15 & 1,23 & 1,32 & 1,35 & 1,20 & 1,44 & 1,09 \\
Coef. Variação & 20,89 & 25,10 & 25,60 & 24,52 & 24,65 & 25,53 & 25,19 & 24,98 & 25,97 & 23,66
\end{tabular}

PM: Penman-Monteith - FAO; Sx: Haregreaves e Samani (1985).

Bebedouro. Para a maioria dos resultados o valor do coeficiente de correlação " $r$ " pode ser considerado de moderado a alto (Hopkins, 2012).

Na Tabela 3 observa-se uma superestimativa de 13\% de Hargreaves-Samani (1985) em relação ao padrão, valor bem abaixo dos relatados na literatura, que descreve discrepâncias de $39,64 \%$ (Batista et al., 2007). O comportamento de superestimativa também foi observado nas demais condições simuladas, alcançando valores máximos de $23 \%$ em S7.

O SEE variou de 1,02 a 1,81 $\mathrm{mm} \mathrm{d}^{-1}$, para S8 e S1, com os valores intermediários bem próximos entre si. Nos valores de SEEa observa-se a melhora após as correções, exceto em S2 e S8, onde a correção não surtiu eficiência na diminuição do erro. A maior correção proporcionada pelo ajuste foi de 0,56 $\mathrm{mm} \mathrm{d}^{-1} \mathrm{em} \mathrm{S} 7 \mathrm{e}$ a menor $0,03 \mathrm{~mm} \mathrm{~d}^{-1} \mathrm{em} \mathrm{S6}$.

Para todos os cenários os valores de concordância foram classificados como muito bom (Camargo e Sentelhas, 1997), com valores variando de 0,7 a 0,8 , semelhante ao relatado por Souza et al. (2011) para o método de Hargreaves e Samani (1985).

\section{CONCLUSÕES}

O método de Hargreaves e Samani (1985) tende a superestimativa dos valores mensais de ETo em até $13 \%$, quando comparado ao método padrão de Penman-Monteith - FAO, destacando-se maior discrepância quando se altera inversamente 

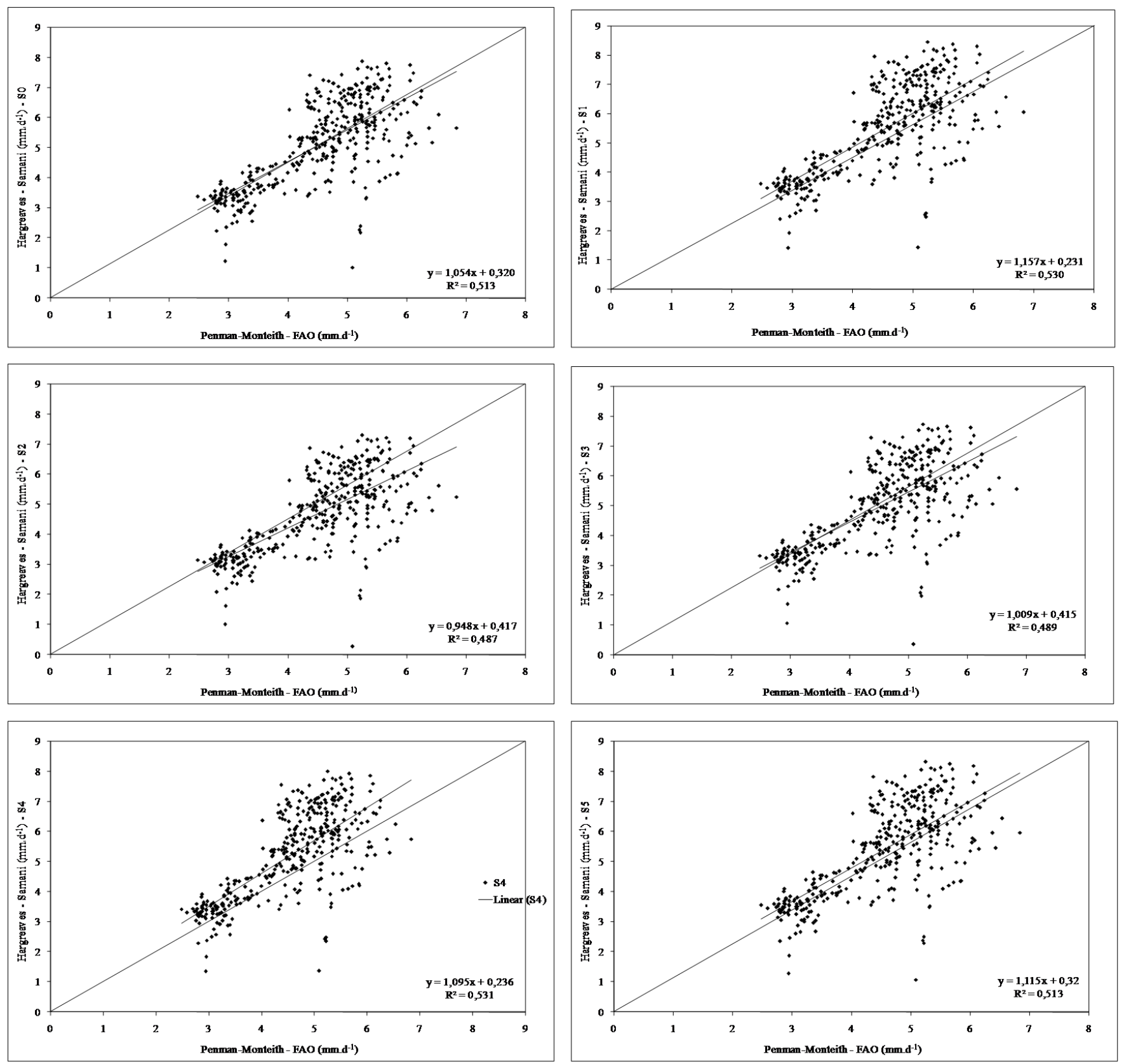

Figura 3 - Relação entre a ETo diária estimada pelo método de Penman-Monteith - FAO e pelo método de Hargreaves-Samani sem e com alterações de $5 \%$ nas temperaturas máximas e mínimas. Bebedouro - SP.

a temperatura máxima e mínima, com superestimativa de $23 \%$, para a região de Bebedouro, SP.

Uma variação de 5\% nas temperaturas máximas e mínimas não inferem em erros grosseiros nas estimativas de ETo utilizando a metodologia proposta por Hargreaves e Samani (1985), com valores não ultrapassando a casa dos $1,03 \mathrm{~mm} \mathrm{dia}^{-1}$, média mensal.

O acréscimo de cinco por cento na temperatura máxima refletiu nas maiores discrepância nos valores estimados de ETo frente ao método padrão, com percentuais de estimativas da ordem de 18,7 a $22,8 \%$. Já na temperatura mínima este reflete superestimativa menor em relação ao padrão.

\section{AGRADECIMENTOS}

Os autores agradecem ao Conselho Nacional de Desenvolvimento Científico e Tecnológico (CNPq) e à Coordenação de Aperfeiçoamento de Pessoal de Nível Superior 

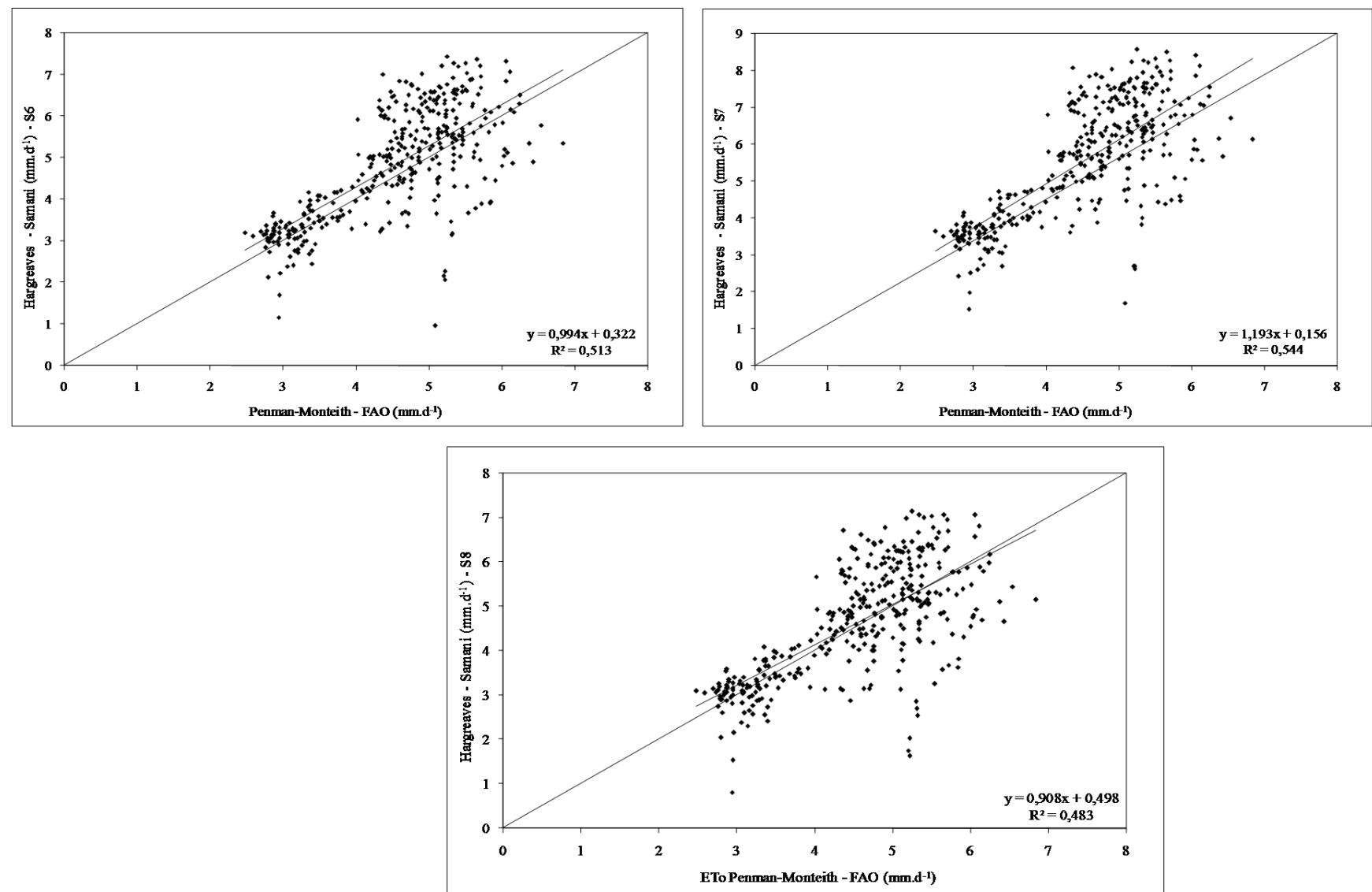

Figura 4 - Relação entre a ETo diária estimada pelo método de Penman-Monteith - FAO e pelo método de Hargreaves-Samani sem e com alterações de $5 \%$ nas temperaturas máximas e mínimas. Bebedouro - SP.

Tabela 3 - Análise das variações em relação ao método padrão: (1) Percentagem de variação; (2) erro-padrão de estimativa (EPE - mm d ${ }^{-1}$ ); (3) erro-padrão de estimação ajustada $\left(\mathrm{EPEa}-\mathrm{mm} \mathrm{d}^{-1}\right)$ e (4) índice de concordância, adimensional.

\begin{tabular}{ccccc|cccccc}
\hline & & & & & & & & & & \\
Método & & & & & & & & \\
& & & & & & & & \\
& 1 & 2 & 3 & 4 & & & & & \\
& & & & & & 2 & 3 & 4 \\
\hline PM & 1,00 & - & - & - & S4 4 & 1,15 & 1,60 & 1,33 & 0,75 \\
S0 & 1,13 & 1,54 & 1,36 & 0,76 & S5 & 1,19 & 1,73 & 1,36 & 0,71 \\
S1 & 1,21 & 1,81 & 1,33 & 0,69 & S6 & 1,07 & 1,38 & 1,35 & 0,80 \\
S2 & 1,04 & 1,33 & 1,65 & 0,80 & S7 & 1,23 & 1,88 & 1,32 & 0,68 \\
S3 & 1,10 & 1,48 & 1,42 & 0,77 & S8 & 1,02 & 1,27 & 1,40 & 0,81 \\
\hline
\end{tabular}


(CAPES) pelo auxílio financeiro e a Estação Experimental de Citricultura de Bebedouro (EECB) pelo aporte institucional e de pessoal.

\section{REFERÊNCIAS BIBLIOGRÁFICAS}

ALLEN, R.G.; PEREIRA, L.S.; RAES, D.; SMITH, M. Guidelines for computing crop water requeriments. Rome: FAO, 1998. 308 p. (FAO - Irrigation and Drainage, 56).

BATISTA, W. R.M.; FACCIOLI, G. G.; SILVA, A. A. G. Determinação e comparação entre métodos de estimativa da evapotranspiração de referência para a região de Canindé do São Francisco-CE. Revista da Fapese, v.3, n. 2, p. 7176, 2007.

CAMARGO, A. P. de; SENTELHAS, P. C. Avaliação do desempenho de diferentes métodos de estimativa da evapotranspiração potencial no Estado de São Paulo, Brasil. Revista Brasileira de Agrometeorologia, Santa Maria, v. 5, n. 1, p. 89 - 97, 1997.

FNP CONSULTORIA \& COMERCIO. Agrianual 2011: Anuário da agricultura brasileira. Sao Paulo, 2011. p. 245-306.

FRANÇA NETO, A.C.F. Análise de métodos simplificados de estimativa da eto e da sensibilidade das variáveis do cálculo da lâmina de irrigação para a cultura do café. 2003. 82 f. Dissertação (Mestrado em Meteorologia Agrícola) Universidade Federal de Viçosa, Viçosa, 2003.

HARGREAVES, G.H.; SAMANI, Z.A. Reference crop evapotranspiration from ambient air temperature. Chicago: American Society of Agricultural and Biological Engineers Meeteng, 1985. (Paper 85 -2517).

HOPKINS, W. G. Correlation Coefficient, 2012. Disponível em: http://www.sportsci.org/resource/stats/correl.html. Acesso em: 14 out. 2012 .

IBGE - Instituto Brasileiro de Geografia e Estatistica. Censo agropecuário - 2006. Rio de Janeiro, 2009.

JENSEN, M.E.; BURMAN, R.D.; ALLEN, R.G. Evapotranspiration and irrigation water requeriments. New York: ASCE, 1990. 332 p.
MANTOVANI, E. C. Desarollo y evaluación de modelos para el manejo del riego: estimación de Ia evapotranspiración y efectos de la uniformidad de aplícación del riego sobre la producción de los cultivos. 1993. 184 f. Tese (Doutorado em Agronomia) - Escuela Ténica Superior de Ingenieros Agrónomos y Montes de la Universidade de Córdoba. Córdoba, 1993.

MELLO, J. P. Análise de sensibilidade dos componentes da equação de Penmam-Monteith-FAO. 1998. 79 f. Tese (Doutorado em Engenharia Agrícola) - Universidade Federal de Viçosa, Viçosa. Viçosa, 1998.

MENDONÇA, J. C. SOUSA, E. F.; BERNARDO, S.; DIAS, G. P.; GRIPPA, S. Comparação entre métodos de estimativa da evapotranspiração de referência (ETo) na região Norte Fluminense, RJ. Revista Brasileira de Engenharia Agrícola e Ambiental, v. 7, n. 2, p. 275-279, 2003.

PEREIRA, A. R.; VILA NOVA, N. A.; SEDIYAMA, G.C. Evapotranspiração. Fundação de Estudos Agrários. Luiz de Queiros: FEALQ, Piracicaba, 183p. 1997.

ROLIM, G.S. et al . Classificação climática de Köppen e de Thornthwaite e sua aplicabilidade na determinação de zonas agroclimáticas para o estado de são Paulo. Bragantia, Campinas, v. 66, n. 4, 2007.

SOUZA, A. P. de et al . Estimativas da evapotranspiração de referência em diferentes condições de nebulosidade. Pesquisa Agropecuária, 2011. Disponível em: http://www. scielo.br/scielo.php?script=sci arttext\&pid=S0100204X201 1000300001\&lng=pt\&nrm=iso. Acesso em: 20 dez. 2012.

VEGA, E.C.; JARA, J.C. Estimación de la evapotranspiración de referencia para dos zonas (costa y región andina) del Ecuador. Engenharia Agrícola, Jaboticabal, v.29, n.3, p.390-403, 2009.

WILlMOTT, C.J.; CKLESON, S.G.; DAVIES, R.E. Statistics for evaluation and comparisons of models. Journal of Geophysical Research, v.90, n. C5, p. 8995-9005, 1985. 